\title{
Reduced Order Model and Robust Control Architecture for Mechanical Systems with Nonholonomic Pfaffian Constraints
}

\author{
Chun-Yi Su, Member, IEEE, Yury Stepanenko, and A. A. Goldenberg, Fellow, IEEE
}

\begin{abstract}
The position/force control of mechanical systems subject to a set of Pfaffian constraints is addressed in this paper. A reduced order dynamical description of such nonholonomic mechanical systems, including the constraints, is developed. Some properties of the dynamic model are then exploited to facilitate the controller design. Based on theory of guaranteed stability of uncertain systems, a robust control algorithms is derived, guaranteeing the uniform ultimate boundedness of the tracking errors. A detailed numerical example is presented to illustrate the developed method.
\end{abstract}

Index Terms-Mechanical systems, nonholonomic constraints, robust control.

\section{INTRODUCTION}

$\mathbf{I}^{\mathrm{N}}$ $\mathrm{N}$ RECENT years, the control of mechanical systems with nonholonomic constraints is a subject that has generated significant interest for numerous practical applications. It is well known that in mobile robots, multifingered hands manipulating a grasped object, and space robots, the kinematic constraint equations are nonholonomic [9], and the dynamics of such systems are well understood (see, e.g., [9], [13]). Control of mechanical systems, with nonholonomic constraints, has been described through the special cases of mobile wheeled robots in [14], [15], [18], [19]. Early work that deals with control of nonholonomic systems is described in [8]. However, a theoretic framework which can serve as a basis for the study of mechanical systems with nonholonomic constraints is quite recent [1], [2], [4], [5]. Nevertheless, all of those discussions are only focused on a special case (Pfaffian catastatic constraints) [10].

As discussed in [9], [10], different types of constraints may occur in different physical contests. The constraints for coupled rigid bodies, for example, is different from the constraints occurred in rolling and cutting motions [7], [12]. It is known that nonholonomic Pfaffian constraints represent a broad class of constraints [10]. An example which illustrates the Pfaffian constraints is a homogeneous ball on a rotating plate [11]. In this paper, our attention is focused on the

Manuscript received August 8, 1997; revised November 8, 1998. This work was supported by the Natural Science and Engineering Research Council of Canada, the Institute for Robotic and Intelligent Systems (IRIS), and Precarn Associates, Inc.

C.-Y. Su is with the Department of Mechanical Engineering, Concordia University, Montreal, P.Q., H3G 1M8, Canada.

Y. Stepanenko is with the Department of Mechanical Engineering, University of Victoria, Victoria, B.C., V8W 3P6, Canada.

A. A. Goldenberg is with Department of Mechanical Engineering, University of Toronto, Ont., M5S 1A4, Canada.

Publisher Item Identifier S 1083-4427(99)03330-5. general Pfaffian constraints, and analyses are given from the Lagrangian point of view. As for the Hamiltonian case with other forms of nonholonomic constraints, the reader may refer to [3]. By assuming complete knowledge of the constraint manifold, and taking the internal dynamics of the systems into account due to nonholonomic constraints, a reduced order dynamics description suitable for motion and force control is derived. Then by exploiting the particular structure of the dynamics, several fundamental properties are obtained to facilitate the controller design. Finally, with the specification of a desired manifold, a robust control algorithm is derived, where the control of the constraint force due to the existence of Pfaffian constraints is also included, using only the measurements of joint position, velocity, and constraint force. Stability analysis shows the stabilization of the manifold in the sense that tracking errors are uniformly ultimately bounded.

\section{DYNAMIC EQUATION WITH PFAFFIAN CONSTRAINTS}

In this section, we consider a mechanical system with $n$ degree-of-freedom whose generalized coordinates are $q_{1}, q_{2}, \cdots, q_{n}$. The Lagrange equations describing the motion of the system are

$$
\frac{d}{d t}\left(\frac{\partial \mathcal{L}}{\partial \dot{q}_{r}}\right)-\frac{\partial \mathcal{L}}{\partial q_{r}}=\tau_{r}, \quad r=1,2, \cdots, n
$$

where $\mathcal{L}=T-P, T$ and $P$ are the kinetic and potential energy respectively, and $\tau_{r}$ is the generalized force. The kinetic energy can be expressed as [10], [13]

$$
T=\frac{1}{2} \sum_{i, j=1}^{n} a_{i j} \dot{q}_{i} \dot{q}_{j}
$$

where $a_{i j}$ is a function of the generalized coordinates.

Let us consider the situation where the system is subjected to additional $p(p<n)$ independent nonintegrable kinematic constraints of the form [10], [13]

$$
\sum_{r=1}^{n} \iota_{k r} d q_{r}+\beta_{k l} d t=0, \quad k=1,2, \cdots, p
$$

where $\iota_{k r}$ and $\beta_{k l}$ are (at least once piecewise differentiable) functions of the generalized coordinates and time. The constraint equation (3) is called Pfaffian form [10]. When $\beta_{k l} \equiv 0$, these constraints are called catastatic, otherwise they are acatastatic [10]. The geometric interpretation of catastatic and acatastatic constraints is illustrated in [10]. In the following development, we will focus on the general form of (3). It should be noted that since (3) is nonintegrable, one 
cannot solve for $p$ of the variables in $q_{r}(r=1, \cdots n)$ in terms of those remaining to reduce its dimension through direct substitution into (1). It is important to note that the constraints (3) is nonintegrable. Otherwise, the constraints can be integrated into holonomic constraints and in this case they can be used to reduce the system dimension by eliminating some generalized coordinates. The conditions for checking this nonintegrability are given in [22].

When the nonholonomic constraints (3) are imposed on the mechanical systems (1), these constraints may be thought of as imposing additional constraint forces, $\tau_{r}^{\prime}$, on the system, thereby altering the set of (1) to [10]

$$
\frac{d}{d t}\left(\frac{\partial \mathcal{L}}{\partial \dot{q}_{r}}\right)-\frac{\partial \mathcal{L}}{\partial q_{r}}=\tau_{r}+\tau_{r}^{\prime}, \quad r=1,2, \cdots, n
$$

where

$$
\tau_{r}^{\prime}=\sum_{k=1}^{p} \lambda_{k} \iota_{k r}
$$

and quantities $\lambda_{k}(k=1, \cdots, p)$ are Lagrangian multipliers [9].

In the following, we denote $\boldsymbol{q}=\left[\begin{array}{ll}q_{1} & q_{2} \cdots q_{n}\end{array}\right]^{T}$, then Lagrange's equation (4) can be manipulated to derive

$$
D(\boldsymbol{q}) \ddot{\boldsymbol{q}}+F(\dot{\boldsymbol{q}}, \boldsymbol{q}) \dot{\boldsymbol{q}}+G(\boldsymbol{q})=\tau+L^{T} \lambda
$$

where the $n \times n$ matrix $D(q)$ is positive definite and symmetric, and is related to the inertial properties of system [10], the vector function $F(\dot{\boldsymbol{q}}, \boldsymbol{q}) \dot{\boldsymbol{q}}$ is in general a nonlinear function of its arguments and $\tau=\left[\tau_{1}, \tau_{2}, \cdots \tau_{n}\right]^{T}, L$ is denoted by the $p \times n$ matrix $[\ell]_{i j}$, and $\lambda=\left[\lambda_{1}, \cdots \lambda_{p}\right]^{T}$ is the associated Lagrangian multipliers.

In the following development we only consider the systems with the following two simplifying properties.

Property 1: A suitable definition of $F(\boldsymbol{q}, \dot{\boldsymbol{q}})$ makes the matrix $(\dot{D}-2 F)$ skew-symmetric. In particular, this is true if the elements of $F(\boldsymbol{q}, \dot{\boldsymbol{q}})$ are defined as

$$
F_{i j}=\frac{1}{2}\left[\dot{\boldsymbol{q}}^{T} \frac{\partial D_{i j}}{\partial \boldsymbol{q}}+\sum_{k=1}^{n}\left(\frac{\partial D_{i k}}{\partial q_{j}}-\frac{\partial D_{j k}}{\partial q_{i}}\right) \dot{q}_{k}\right] .
$$

Property 2: There exists a $m$-vector $\boldsymbol{\alpha}$ with components depending on mechanical parameters (masses, moments of inertia, etc.), such that

$$
D(\boldsymbol{q}) \ddot{\boldsymbol{q}}+F(\boldsymbol{q}, \dot{\boldsymbol{q}}) \dot{\boldsymbol{q}}+G(\boldsymbol{q})=\Phi(\boldsymbol{q}, \dot{\boldsymbol{q}}, \ddot{\boldsymbol{q}}) \alpha
$$

where $\Phi$ is a $n \times m$ matrix of known functions of $\boldsymbol{q}, \dot{\boldsymbol{q}}, \ddot{\boldsymbol{q}}$; and $\alpha$ is the $m$-vector of inertia parameters.

It should be noted that the first property is related to the passivity of the mechanical dynamics and the second property says that the Lagrangian dynamic equation are linearly parameterizable.

The constraints (3) can be written in matrix form as

$$
L \dot{\boldsymbol{q}}(t)=W(\boldsymbol{q}(t), t)
$$

where, as in (5), we have denoted by $L$ the $p \times n$ matrix $[\iota]_{i j}$ and by $W$ the vector containing the remainder of the terms in (3). As in [1], we assume that the matrix $L$ has full rank $p$, which means that the number of constraint equations (3) is unchanged for all $q \in \Re^{n}$ and $t \in \Re$.
Performing a $Q R$-like decomposition of the constraint matrix $L(\boldsymbol{q})$, one obtains

$$
\begin{aligned}
L^{T}(\boldsymbol{q}) & =Q(\boldsymbol{q}) R(\boldsymbol{q}) \\
& =\left[Q_{1}(\boldsymbol{q}) Q_{2}(\boldsymbol{q})\right]\left[\begin{array}{c}
R_{1}(\boldsymbol{q}) \\
0
\end{array}\right] \\
& =Q_{1}(\boldsymbol{q}) R_{1}(\boldsymbol{q})
\end{aligned}
$$

where $Q(\boldsymbol{q}) \in \Re^{n \times n}, R_{1}(\boldsymbol{q}) \in \Re^{p \times p}$ are nonsingular; the columns of $Q_{1}$ are orthonormal and are constructed from $p$ linearly independent columns of $L^{T}$ so that $\mathcal{R}\left(Q_{1}\right)=\mathcal{R}\left(L^{T}\right)$, the columns of $Q_{2}$ are chosen from the orthogonal complement of $R\left(Q_{1}\right)$ so that $\mathcal{R}\left(Q_{2}\right)=\mathcal{N}(L)$. Then we have

$$
\Re^{n}=\mathcal{R}\left(Q_{1}\right) \oplus \mathcal{R}\left(Q_{2}\right) .
$$

Note that in this paper, $\mathcal{R}(\cdot), \mathcal{N}(\cdot)$ denote range and null spaces, respectively.

Based on the above decomposition, the following property could be obtained.

Property 3:

$$
\begin{aligned}
Q_{1}^{T}(\boldsymbol{q}) Q_{1}(\boldsymbol{q}) & =I_{p} \\
Q_{1}^{T}(\boldsymbol{q}) Q_{2}(\boldsymbol{q}) & =0 \\
L(\boldsymbol{q}) Q_{2}(\boldsymbol{q}) & =0 .
\end{aligned}
$$

In the following development, we assume that there exists a minor of $L(\boldsymbol{q})$ having the same rank of $L(\boldsymbol{q})$ for all values of $\boldsymbol{q}$, so that Property 3 is valid globally. In view of the decomposition (10), the constraint equations (9) can be rewritten as

$$
Q_{1}^{T}(\boldsymbol{q}) \dot{\boldsymbol{q}}(t)=R_{1}^{-T}(\boldsymbol{q}) W(\boldsymbol{q}(t), t) .
$$

It can easily be seen that the general solution to (9) or equivalently (14) can then be expressed as

$$
\begin{aligned}
\dot{\boldsymbol{q}} & =Q_{2}(\boldsymbol{q}) \dot{\boldsymbol{v}}^{a}+Q_{1}(\boldsymbol{q}) R_{1}^{-T}(\boldsymbol{q}) W(\boldsymbol{q}(t), t) \\
& =Q_{2}(\boldsymbol{q}) \dot{\boldsymbol{v}}^{a}+H(\boldsymbol{q}, t)
\end{aligned}
$$

where $H(\boldsymbol{q}, t)=Q_{1}(\boldsymbol{q}) R_{1}^{-T}(\boldsymbol{q}) W(\boldsymbol{q}(t), t)$ and $\dot{\boldsymbol{v}}^{a} \in \Re^{n-p}$ is an arbitrary vector, which will be explained later. It should be noted that the first part of the solution (15) is the homogeneous solution lying in $\mathcal{N}\left(Q_{1}^{T}\right)$ and the other is a particular solution to (9). $\dot{v}^{a} \in \Re^{n-p}$ can be thought of as the magnitude of generalized velocity along tangent directions defined by columns of $Q_{2}$.

In (15), a new vector $\boldsymbol{v}^{a} \in \Re^{n-p}$ is introduced and constitute a reduced set of generalized velocities. We should note that similar vectors have also been introduced, for example, in [20], [7] (acatastatic constraints) and [2], [6] (catastatic constraints), though different methods are used. Since $\dot{v}^{a}$ is the same with that in [6] if $W(\boldsymbol{q}(t), t)=0$, we also call $\dot{\boldsymbol{v}}^{a}$ as pseudovelocities. We should note that choice of $\dot{v}^{a}$ is related to the form of constraint equations (9) and may or may not be a physical quantity (see the simulation example). In this paper, we assume that there exists a vector of $\boldsymbol{v}^{a}(\boldsymbol{q}) \in \Re^{n-p}$ so that $\dot{\boldsymbol{v}}^{a}$ is the derivative of $\boldsymbol{v}^{a}$. Without losing generality, we also assume that the components of $\boldsymbol{v}^{a}$ are smooth, $\boldsymbol{v}^{a}(0)=0$, and the set $\left\{\boldsymbol{d} v_{1}^{a}(\boldsymbol{q}), \cdots, \boldsymbol{d} v_{n-p}^{a}(\boldsymbol{q})\right\}$ is linearly independent for all $\boldsymbol{q} \in \Re^{n}$, where $\boldsymbol{d} v_{i}^{a}(\boldsymbol{q})$ is defined as the gradient of $v_{i}^{a}(\boldsymbol{q})$. Choosing $p$ out of $n$ coordinates $\boldsymbol{q}$, denoted by

$$
\boldsymbol{v}^{b}=\left[v_{1}^{b}(\boldsymbol{q}) \cdots v_{p}^{b}(\boldsymbol{q})\right]^{T}
$$


such that the set $\left\{\boldsymbol{d} v_{1}^{a}(\boldsymbol{q}), \cdots, \boldsymbol{d} v_{n-p}^{a}(\boldsymbol{q}), \boldsymbol{d} v_{1}^{b}(\boldsymbol{q}), \cdots, \boldsymbol{d} v_{p}^{b}(\boldsymbol{q})\right\}$ is linearly independent for all $\boldsymbol{q} \in \Re^{n}$. Therefore, for $\boldsymbol{v}^{T}=$ $\left[\boldsymbol{v}^{a T} \boldsymbol{v}^{b T}\right]^{T}$ there exist a global diffeomorphism

$$
\boldsymbol{v}=T(\boldsymbol{q}), T(0)=0, \boldsymbol{v} \in \Re^{n}
$$

which changes coordinates form the basis of $\boldsymbol{q}$ to the basis of $\boldsymbol{v}$. It should be noted that the expression of the diffeomorphism $T$ depends on the form of constraint equations (9) and the choices of $\boldsymbol{v}^{b}$.

Due to (17) and (15), the state $(\boldsymbol{q}, \dot{\boldsymbol{q}})$ can be replaced by $\left(\boldsymbol{v}, \dot{\boldsymbol{v}}^{a}\right)$, i.e., $\left(\boldsymbol{v}, \dot{\boldsymbol{v}}^{a}\right)$ is sufficient to describe the constrained motion. Since $\boldsymbol{q}$ and $\dot{\boldsymbol{q}}$ are measurable directly, in the following development we still use $\boldsymbol{q}$ and $\dot{\boldsymbol{q}}$ as arguments in $D, F$, etc. However, we should remember that $\boldsymbol{q}$ and $\dot{\boldsymbol{q}}$ are related to $\boldsymbol{v}$ and $\boldsymbol{v}^{a}$ through (17) and (15). Differentiating (15), we obtain

$$
\ddot{\boldsymbol{q}}=Q_{2}(\boldsymbol{q}) \ddot{\boldsymbol{v}}^{a}+\frac{d}{d t}\left(Q_{2}(\boldsymbol{q})\right) \dot{\boldsymbol{v}}^{a}+\frac{d}{d t} H(\boldsymbol{q}, t) .
$$

Therefore, the dynamic equation (4), when satisfying the nonholohomic constraint (3), can be expressed in terms of states $\dot{\boldsymbol{v}}^{a}$ as

$$
\begin{aligned}
& D(\boldsymbol{q}) Q_{2}(\boldsymbol{q}) \ddot{\boldsymbol{v}}^{a}+F_{1}(\dot{\boldsymbol{q}}, \boldsymbol{q}) \dot{\boldsymbol{v}}^{a}+G(\boldsymbol{q})+P(\boldsymbol{q}, \dot{\boldsymbol{q}}, t) \\
& \quad=\boldsymbol{\tau}+L^{T}(\boldsymbol{q}) \lambda
\end{aligned}
$$

where

$$
\begin{aligned}
F_{1}(\dot{\boldsymbol{q}}, \boldsymbol{q}) & =D(\boldsymbol{q}) \frac{d}{d t}\left(Q_{2}(\boldsymbol{q})\right)+F(\dot{\boldsymbol{q}}, \boldsymbol{q}) Q_{2}(\boldsymbol{q}) \\
P(\boldsymbol{q}, \dot{\boldsymbol{q}}, t) & =D(\boldsymbol{q}) \frac{d}{d t} H(\boldsymbol{q}, t)+F(\dot{\boldsymbol{q}}, \boldsymbol{q}) H(\boldsymbol{q}, t) .
\end{aligned}
$$

It should be noted that the reduced state space is $2 n-p$ dimensional. The system can be described by the $n$-vector of variables $\boldsymbol{v}$ [or $\boldsymbol{q}$ due to (17)] and the $(n-p)$-vector of variables $\dot{\boldsymbol{v}}^{a}$.

Remark: We note the derived equations for the system include the effects of the Pfaffian constraints. They can therefore be thought of as the equivalent equations of motion. A possible disadvantage of the present formulation is that $\dot{\boldsymbol{v}}^{a} \in \Re^{n-p}$ may not be a physical quantity. Using similar definitions of $\dot{\boldsymbol{v}}^{a}$, various reduced order models have also been obtained in [7], [20] (acatastatic constraint case) and [2], [6] (catastatic constraint case). As will become clear later, in this paper we do not aim at linearizing the system dynamics as, for example, in [7], but instead exploit the physical structure of the systems for the subsequent development.

By exploiting the structure of (19), three properties are obtained.

Property 4: The matrix $Q_{2}^{T} D(\boldsymbol{q}) Q_{2}$ is symmetric and positive definite.

Remark: Property 4 can be easily obtained by observing that $Q_{2}(\boldsymbol{q})$ is of full column rank and $D(\boldsymbol{q})$ is positive definite.

Property 5: Define $D_{1}(\boldsymbol{q})=Q_{2}^{T} D(\boldsymbol{q}) Q_{2}$. Using the same definition of the matrix $F(\dot{\boldsymbol{q}}, \boldsymbol{q})$ as in Property $1, D_{1}(\boldsymbol{q})$ and $F_{1}(\dot{\boldsymbol{v}}, \boldsymbol{q})$ satisfy

$$
\boldsymbol{x}^{T}\left(\frac{d}{d t} D_{1}(\boldsymbol{q})-2 Q_{2}^{T} F_{1}(\dot{\boldsymbol{q}}, \boldsymbol{q})\right) \boldsymbol{x}=0
$$

i.e., $\left((d / d t) D_{1}(\boldsymbol{q})-2 Q_{2}^{T} F_{1}(\dot{\boldsymbol{q}}, \boldsymbol{q})\right)$ is a skew symmetric matrix.

Proof: Directly, by using the definition of $\dot{D}_{1}$ and $F_{1}$ and by considering the skew symmetry of $(\dot{D}-2 F)$ in Property 2 .
Property 6: The dynamic structure (19) is linear in terms of the same suitably selected set of inertia parameters as used in Property 2

$$
\begin{aligned}
& D(\boldsymbol{q}) Q_{2}(\boldsymbol{q}) \ddot{\boldsymbol{v}}^{a}+F_{1}(\boldsymbol{q}, \dot{\boldsymbol{q}}) \dot{\boldsymbol{v}}^{a}+G(\boldsymbol{q})+P(\boldsymbol{q}, \dot{\boldsymbol{q}}, t) \\
& \quad=\Phi_{1}\left(\boldsymbol{q}, \dot{\boldsymbol{q}}, \dot{\boldsymbol{v}}^{a}, \ddot{\boldsymbol{v}}^{a}, t\right) \alpha
\end{aligned}
$$

where $\Phi_{1}$ is a $(n \times m)$ regressor matrix; $\alpha$ is the $m$-vector of inertia parameters.

Remark: Property 6 may be easily understood by observing that the transformations do not change the linearity in terms of constant parameters $\alpha$, established for model (6) by Property 2 .

The aforementioned properties are fundamental for designing the force/motion control law.

\section{Robust CONTROLleR Design FOR MOTION/FORCE TRACKING}

It has been proved (see [1] and [8]) that the nonholonomic systems cannot be stabilized to a single point using pure smooth state feedback. It can only be stabilized to a manifold of dimension $n-p$ due to the existence of $p$ nonholonomic constraints. The objective of stabilizing the systems to a point has been achieved by open-loop control [5], nonsmooth feedback law [1], [18] and time-varying feedback laws [15]-[17]. However, it is fair to say that these approaches are not yet fully general and only focused on the catastatic constraints. It is worth mentioning that different control objectives may also be pursued, such as stabilization to manifolds of equilibrium points [1], [2], [4] (as opposed to a single equilibrium position).

By assuming that variables $\boldsymbol{v}^{a}$ are physically motivated, the objective of the control can be specified as: given a desired $\boldsymbol{v}_{d}^{a}, \dot{\boldsymbol{v}}_{d}^{a}$, and desired constraint force $\tau_{d}^{\prime}$, or identically desired multiplier $\lambda_{d}$, determine a control law such that for any $(\boldsymbol{q}(0), \dot{\boldsymbol{q}}(0)) \in \Omega$, then $\boldsymbol{v}^{a}, \dot{\boldsymbol{v}}^{a}$, and $\lambda$ asymptotically converge to a manifold $\Omega_{d}$ defined as

$$
\Omega_{d}=\left\{(\boldsymbol{q}, \dot{q}, \lambda) \mid \boldsymbol{v}^{a}=\boldsymbol{v}_{d}^{a}, \dot{\boldsymbol{v}}^{a}=\dot{\boldsymbol{v}}_{d}^{a}, \lambda=\lambda_{d}\right\} .
$$

Now, assume that the parameters of the mechanical systems are not exactly known. To synthesize the robust controller, we define

$$
\begin{aligned}
& e_{v}=v^{a}-v_{d}^{a} \\
& \boldsymbol{e}_{\lambda}=\lambda-\lambda_{d} \\
& \dot{v}_{r}=\dot{v}_{d}^{a}-\Lambda \boldsymbol{e}_{\boldsymbol{v}}
\end{aligned}
$$

where $\Lambda$ is a positive definite matrix whose eigenvalues are strictly in the right-hand complex plane.

Before giving the control law, the following assumptions are required.

Assumption A1: The desired trajectory $\boldsymbol{v}_{d}^{a}$ is chosen such that $\boldsymbol{v}_{d}^{a}, \dot{\boldsymbol{v}}_{d}^{a}$, and $\ddot{\boldsymbol{v}}_{d}^{a}$ are all bounded signals.

Assumption A2: $D(\boldsymbol{q}), F(\dot{\boldsymbol{q}}, \boldsymbol{q})$, and $G(\boldsymbol{q})$ are bounded in $\boldsymbol{q}$, and $F(\dot{\boldsymbol{q}}, \boldsymbol{q})$ is linear in $\dot{\boldsymbol{q}}$, therefore, there exist positive constants $\kappa_{i}, i=1,2,3$, such that

$$
\begin{aligned}
\|D(\boldsymbol{q})\| & \leq \kappa_{1} \\
\|F(\dot{\boldsymbol{q}}, \boldsymbol{q})\| & \leq \kappa_{2}\|\dot{\boldsymbol{q}}\| \\
\|G(\boldsymbol{q})\| & \leq \kappa_{3}
\end{aligned}
$$


where the norm of vector $x$ is defined as $\|x\|=\sum_{i=1}^{n}\left|x_{i}\right|$ and that of matrix $A$ is defined as the corresponding induced norm. Throughout this paper, we adopt this definition for the norm.

Assumption A3: The matrix $D_{1}(\boldsymbol{q})=Q_{2}^{T} D(\boldsymbol{q}) Q_{2}$ can be bounded above and below, i.e.,

$$
\beta_{m} I \leq D_{1}(\boldsymbol{q}) \leq \beta_{M} I \quad \forall \boldsymbol{q} \in \Re^{n}
$$

where $\beta_{m}$ and $\beta_{M}$ are positive constants.

Remark: The remarks about this set of assumptions are worth making at this juncture. Assumption A1 is common to most tracking schemes. Assumption A2 is valid for a wide class of mechanical systems, for example, robots with revolute joints. Assumption A2 can be extended to a more general case. However, our goal in this paper is to develop the new control strategy in a simpler setting that reveals its essential features. This is the motivation for simply stating it as an assumption. Also, the exact values of $\kappa_{i}(i=1,2,3)$ are not necessary for the controller design. As for Assumption A3, since the matrix $D(\boldsymbol{q})$ is always bounded above and below, and $Q_{2}(\boldsymbol{q})$ depend only on the constraint equation (9), Assumptions A3 can always be satisfied for a class of Pfaffian constraints.

Defining $\alpha$ as a $m$-vector, containing the unknown elements in the suitably selected set of equivalent dynamic parameters, then the linear parameterizability of the dynamics (Property 6) leads to

$$
\begin{aligned}
& D(\boldsymbol{q}) Q_{2}(\boldsymbol{q}) \ddot{\boldsymbol{v}}_{r}+F_{1}(\boldsymbol{q}, \dot{\boldsymbol{q}}) \dot{\boldsymbol{v}}_{r}+G(\boldsymbol{q})+P(\boldsymbol{q}, \dot{\boldsymbol{q}}, t) \\
& =\Phi_{1}\left(\boldsymbol{q}, \dot{\boldsymbol{q}}, \dot{\boldsymbol{v}}_{r}, \ddot{\boldsymbol{v}}_{r}\right) \alpha
\end{aligned}
$$

where $\Phi_{1}$ is the $(n \times m)$ regressor matrix.

The robust control law is then synthesized as

$$
\begin{aligned}
& \tau=\Phi_{1}\left(\boldsymbol{q}, \dot{\boldsymbol{q}}, \dot{\boldsymbol{v}}_{r}, \ddot{\boldsymbol{v}}_{r}\right) \varphi-K Q_{2} \boldsymbol{s}-L^{T} \lambda_{c} \\
& \varphi=\left\{\begin{array}{cc}
-\rho \frac{\Phi_{1}^{T} Q_{2} s}{\left\|\Phi_{1}^{T} Q_{2} s\right\|}, & \text { if }\left\|\Phi_{1}^{T} Q_{2} \boldsymbol{s}\right\|>\epsilon \\
-\frac{\rho}{\epsilon} \Phi_{1}^{T} Q_{2} \boldsymbol{s}, & \text { if }\left\|\Phi_{1}^{T} Q_{2} \boldsymbol{s}\right\| \leq \epsilon
\end{array}\right.
\end{aligned}
$$

where $\Phi_{1}$ is defined in (25); $Q_{2}$ is defined in (10); $K$ is an $n \times n$ positive definite matrix, $\rho \in R_{+}$used in (27) is the upper bounds of inertia parameter $\alpha$, i.e., $\|\alpha\| \leq \rho$, which is assumed known; $\epsilon$ is an constant; the vector $\boldsymbol{s}$, which can be thought of as a sliding surface, is defined as

$$
\boldsymbol{s}=\dot{\boldsymbol{e}}_{v}+\Lambda \boldsymbol{e}_{v}
$$

the force term $\lambda_{c}$ is defined as

$$
\lambda_{c}=\lambda_{d}-K_{\lambda} e_{\lambda}
$$

where $K_{\lambda}$ is a constant force control feedback gain.

Based on the above, the following theorem can be stated.

Theorem: Consider the constrained mechanical system (19) with the control laws (26), (27), satisfying Assumptions A1-A3. Then the following holds for any $(q(0), \dot{\boldsymbol{q}}(0)) \in \Omega$ :

1) $\dot{\boldsymbol{e}}_{v}$ and $\boldsymbol{e}_{v}$ are uniformly ultimately bounded.

2) $\boldsymbol{e}_{\lambda}$ is uniformly ultimately bounded and inversely proportional to $\left(1+K_{\lambda}\right)$.

Proof: From the dynamic equation (19) and relationships (28), using (25) and (26) after some calculations, the following is obtained:

$$
D Q_{2} \dot{s}=\Phi_{1} \varphi-\Phi_{1} \alpha-K Q_{2} s-F_{1} s-L^{T}\left(\lambda_{c}-\lambda\right) .
$$

According to Property 3, the above equation becomes

$Q_{2}^{T} D Q_{2} \dot{s}=Q_{2}^{T} \Phi_{1} \varphi-Q_{2}^{T} \Phi_{1} \alpha-Q_{2}^{T} K Q_{2} s-Q_{2}^{T} F_{1} s$.

Thus, we define a Lyapunov function candidate for system (31) as

$$
V=\frac{1}{2} \boldsymbol{s}^{T} D_{1} \boldsymbol{s} .
$$

A simple calculation shows that along solutions of (31)

$$
\begin{aligned}
\dot{V}= & \boldsymbol{s}^{T}\left(Q_{2}^{T} \Phi_{1} \varphi-Q_{2}^{T} \Phi_{1} \alpha-Q_{2}^{T} K Q_{2} \boldsymbol{s}\right) \\
& +\boldsymbol{s}^{T}\left(\frac{1}{2} \dot{D}_{1}-Q_{2}^{T} F_{1}\right) \boldsymbol{s} \\
= & -\boldsymbol{s}^{T} Q_{2}^{T} K Q_{2} \boldsymbol{s}+\boldsymbol{s}^{T} Q_{2}^{T} \Phi_{1}(\varphi-\alpha)
\end{aligned}
$$

where we have used Property 5 to eliminate the term $\boldsymbol{s}^{T}\left(\frac{1}{2} \dot{D}_{1}-Q_{2}^{T} F_{1}\right) \boldsymbol{s}$. Using an argument similar to [21], if $\left\|\Phi_{1}^{T} Q_{2} s\right\|>\epsilon$, the second term in (33) is

$$
\begin{aligned}
& \left(\Phi_{1}^{T} Q_{2} s\right)^{T}(\varphi-\alpha) \\
& \quad=\left(\Phi_{1}^{T} Q_{2} s\right)^{T}\left(-\alpha-\rho \frac{\Phi_{1}^{T} Q_{2} s}{\left\|\Phi_{1}^{T} Q_{2} s\right\|}\right) \\
& \quad \leq\left\|\Phi_{1}^{T} Q_{2} s\right\|(\|\alpha\|-\rho) \leq 0
\end{aligned}
$$

from the Cauchy-Schwartz inequality and our assumption on $\|\alpha\|$. If $\left\|\Phi_{1}^{T} Q_{2} s\right\| \leq \epsilon$, we have

$$
\begin{aligned}
& \left(\Phi_{1}^{T} Q_{2} s\right)^{T}(\varphi-\alpha) \\
& \quad \leq\left(\Phi_{1}^{T} Q_{2} s\right)^{T}\left(\rho \frac{\Phi_{1}^{T} Q_{2} s}{\left\|\Phi_{1}^{T} Q_{2} s\right\|}+\varphi\right) \\
& \quad=\left(\Phi_{1}^{T} Q_{2} s\right)^{T}\left(\rho \frac{\Phi_{1}^{T} Q_{2} s}{\left\|\Phi_{1}^{T} Q_{2} s\right\|}-\frac{\rho}{\epsilon} \Phi_{1}^{T} Q_{2} s\right) .
\end{aligned}
$$

The last term achieves a maximum value of $\epsilon / 2 \rho$ when $\left\|\Phi_{1}^{T} Q_{2} \boldsymbol{s}\right\|=\epsilon / 2$. Thus we have that

$$
\begin{aligned}
\dot{V} & \leq-s^{T} Q_{2}^{T} K Q_{2} s+\epsilon / 2 \rho \\
& \leq-\eta\|s\|^{2}+\delta
\end{aligned}
$$

where $\eta=\lambda_{\min }\left(Q_{2}^{T} K Q_{2}\right), \delta=\epsilon / 2 \rho$. From above we can obtain a larger upper bound of $\boldsymbol{s}$ as

$$
\|\boldsymbol{s}\| \leq\left[\frac{\beta_{M}}{\beta_{m}}\|\boldsymbol{s}(0)\|^{2} e^{-2 \bar{\eta} t}+\frac{\delta}{\bar{\eta} \beta_{m}}\left[1-e^{-2 \bar{\eta} t}\right]\right]^{1 / 2}
$$

where $\bar{\eta}=\eta / \beta_{M}$, and $\beta_{m}$ and $\beta_{M}$ are positive scalars defined in Assumption A3. Therefore, $\boldsymbol{s}$ is uniformly ultimately bounded. By standard linear control arguments and the definition of $\boldsymbol{s}$ in (28), it can be shown that $\boldsymbol{e}_{v}$ and $\dot{\boldsymbol{e}}_{v}$ are also uniformly ultimately bounded.

Since $s, \dot{\boldsymbol{e}}_{\boldsymbol{v}}$, and $\boldsymbol{e}_{v}$ are bounded, it follows that $\dot{\boldsymbol{v}}^{a}, \ddot{\boldsymbol{v}}^{a}, \dot{v}_{r}$, and $\ddot{v}_{r}$ are all bounded. Based on Assumption A2, one can conclude that all signals on the right side of (31) are bounded. Thus, we obtain that $\dot{s}$ and therefore $\ddot{\boldsymbol{v}}^{a}$ are bounded. Equation (30) can be rewritten as

$$
\begin{aligned}
L^{T}\left(\lambda_{c}-\lambda\right) & =\Phi_{1} \varphi-\Phi_{1} \alpha-K Q_{2} s-F_{1} s-D Q_{2} \dot{s} \\
& =\varphi\left(\boldsymbol{q}, \dot{\boldsymbol{q}}, \dot{\boldsymbol{v}}^{a}, \ddot{\boldsymbol{v}}^{a}, \dot{\boldsymbol{v}}_{r}, \ddot{\boldsymbol{v}}_{r}\right)
\end{aligned}
$$

where $\varphi$ is a bounded function. Thus

$$
L^{T} e_{\lambda}=\left(1+K_{\lambda}\right)^{-1} \varphi
$$

are bounded and can be adjusted by changing the feedback gain $K_{\lambda}$. Thus, the theorem is proved. 


\section{Remarks:}

1) In the theorem, the control law is, in a simple fashion, related to the bounds of inertia parameters $\alpha$ so that the parameter variations in the plant can be taken into account easily.

2) From (37), it is shown how decreasing $\epsilon$ the size of the ball within which $\|\boldsymbol{s}\|$ is ultimately confined. If $\epsilon \rightarrow 0$, then $s \rightarrow 0$ and therefore $e_{v} \rightarrow 0$ and $\dot{e}_{v} \rightarrow 0$, $(\boldsymbol{q}, \dot{\boldsymbol{q}}, \lambda) \rightarrow \Omega_{d}$ exponentially. In such a case, $\varphi$ in (26) becomes $\varphi=\operatorname{sgn}\left(\Phi_{1}^{T} Q_{2} s\right)$, which is a typical sliding mode control law. As a matter of fact, the control law (27) is just a smoothing realization of the switch function $\varphi=\operatorname{sgn}\left(\Phi_{1}^{T} Q_{2} s\right)$ so as to overcome chattering, which is undesirable in practice.

3) Suppose $\varphi$ in (26) is replaced by $\hat{\alpha}$, representing estimation of $\alpha$, and $\hat{\alpha}$ is updated by $\dot{\hat{\alpha}}=-\Gamma \Phi_{1}^{T} Q_{2} s$. This leads to an adaptive control law, similar to the one given in [23]. With this algorithm, the closed loop system is globally convergent. However, the question of whether to use robust control or adaptive control does not have an obvious answer. Clearly the adaptive control is easier to design and would be expected to work better if the uncertainty is large. But, it is known that adaptive control performs poorly in the presence of external disturbance and unmodeled dynamics unless the algorithm is modified. Such a modification will result in a more complicated design comparable to the present robust design.

\section{Simulated EXAMPLE}

For simplicity, consider a two-degree-of-freedom mechanical system described by

$$
\begin{aligned}
& {\left[\begin{array}{ll}
D_{11}\left(q_{2}\right) & D_{12}\left(q_{2}\right) \\
D_{12}\left(q_{2}\right) & D_{22}\left(q_{2}\right)
\end{array}\right]\left[\begin{array}{l}
\ddot{q}_{1} \\
\ddot{q}_{2}
\end{array}\right]} \\
& +\left[\begin{array}{ll}
-F_{12}\left(q_{2}\right) \dot{q}_{2} & -F_{12}\left(q_{2}\right)\left(\dot{q}_{1}+\dot{q}_{2}\right) \\
F_{12}\left(q_{2}\right) \dot{q}_{1} & 0
\end{array}\right]\left[\begin{array}{l}
\dot{q}_{1} \\
\dot{q}_{2}
\end{array}\right] \\
& \quad+\left[\begin{array}{l}
g_{1}\left(q_{1}, q_{2}\right) g \\
g_{2}\left(q_{1}, q_{2}\right) g
\end{array}\right] \\
& =\left[\begin{array}{l}
\tau_{1} \\
\tau_{2}
\end{array}\right]+\left[\begin{array}{c}
0 \\
2+\sin \left(q_{1}\right)
\end{array}\right] \lambda
\end{aligned}
$$

where $D_{11}\left(q_{2}\right)=\left(m_{1}+m_{2}\right) r_{1}^{2}+m_{2} r_{2}^{2}+2 m_{2} r_{1} r_{2} \cos \left(q_{2}\right)$; $D_{12}(\phi)=m_{2} r_{2}^{2}+m_{2} r_{1} r_{2} \cos \left(q_{2}\right) ; D_{22}(\phi)=m_{2} r_{2}^{2}$; $F_{12}(\phi)=m_{2} r_{1} r_{2} \sin \left(q_{2}\right) ; g_{1}(\theta, \phi)=\left(m_{1}+m_{2}\right) r_{1} \cos \left(q_{2}\right)+$ $m_{2} r_{2} \cos \left(q_{1}+q_{2}\right) ; g_{2}(\theta, \phi)=m_{2} r_{2} \cos \left(q_{1}+q_{2}\right)$, and let it be constrained in such a way that the velocity of the second link satisfies

$$
\left(2+\sin \left(q_{1}\right)\right) \dot{q}_{2}=1
$$

which is obviously a Pfaffian nonholonomic constraint.

Then

$$
\begin{aligned}
L^{T} & =\left[\begin{array}{c}
0 \\
2+\sin \left(q_{1}\right)
\end{array}\right] \\
& =\left[\begin{array}{ll}
0 & 1 \\
1 & 0
\end{array}\right]\left[\begin{array}{c}
2+\sin \left(q_{1}\right) \\
0
\end{array}\right] .
\end{aligned}
$$

In this case, (15) becomes

$$
\dot{\boldsymbol{q}}=\left[\begin{array}{l}
1 \\
0
\end{array}\right] \dot{v}+\left[\begin{array}{l}
0 \\
1
\end{array}\right] \frac{1}{2+\sin \left(q_{1}\right)} .
$$

where $v$ is just $q_{1}$ and physically motivated. Thus, (18) becomes

$$
\ddot{q}=\left[\begin{array}{l}
1 \\
0
\end{array}\right] \ddot{v}-\left[\begin{array}{l}
0 \\
1
\end{array}\right] \cos \left(q_{1}\right) \dot{q}_{1} \dot{q}_{2}^{2} .
$$

The constrained dynamic equation (19) can be expressed as

$$
\begin{aligned}
D\left[\begin{array}{l}
1 \\
0
\end{array}\right] & \ddot{v}+F\left[\begin{array}{l}
1 \\
0
\end{array}\right] \dot{v}+G+F\left[\begin{array}{l}
0 \\
1
\end{array}\right] \frac{1}{2+\sin \left(q_{1}\right)} \\
& -D\left[\begin{array}{l}
0 \\
1
\end{array}\right] \cos \left(q_{1}\right) \dot{q}_{1} \dot{q}_{2}^{2} \\
= & {\left[\begin{array}{l}
\tau_{1} \\
\tau_{2}
\end{array}\right]+\left[\begin{array}{c}
0 \\
2+\sin \left(q_{1}\right)
\end{array}\right] \lambda . }
\end{aligned}
$$

The desired manifold $\Omega_{d}$ is chosen as

$\Omega_{d}=\left\{(\boldsymbol{q}, \dot{\boldsymbol{q}}, \lambda) \mid q_{1}=q_{1 d}=v_{d}^{a}, \dot{q}_{1}=\dot{q}_{1 d}=\dot{v}_{d}^{a}, \lambda=\lambda_{d}=10\right\}$

where $v_{d}^{a}$ is chosen as $v_{d}^{a}=-90^{\circ}+52.5(1-\cos (1.26 t))$. In this case, $\dot{q}_{2 d}$ should be $\dot{q}_{2 d}=1 /\left(2+\sin \left(q_{1 d}\right)\right)$.

The robust control law (26) with (27) is used so that $\left[q_{1}, \dot{q}_{1}\right]$ and $\lambda$ approach $\Omega_{d}$.

As in [24], one parameterization of (39) is given by

$$
\begin{aligned}
& \alpha_{1}=\left(m_{1}+m_{2}\right) r_{1}^{2} \\
& \alpha_{2}=m_{2} r_{2}^{2} \\
& \alpha_{3}=m_{2} r_{1} r_{2} .
\end{aligned}
$$

Then, the regressor matrix $\Phi_{1}$ defined in (25) can be written as

$$
\begin{aligned}
\phi_{11}= & \ddot{v}_{r}+\varrho \cos \left(q_{2}\right) \\
\phi_{12}= & \ddot{v}_{r}-\dot{q}_{1} \dot{q}_{2}^{2} \cos \left(q_{1}\right) \\
\phi_{13}= & 2 \cos \left(q_{2}\right) \ddot{v}_{r}-\sin \left(q_{2}\right) \dot{q}_{2} \dot{v}_{r}+\varrho \cos \left(q_{1}+q_{2}\right) \\
& \quad-\sin \left(q_{2}\right) \dot{q}_{2}\left(\dot{q}_{1}+\dot{q}_{2}\right)-\cos \left(q_{2}\right) \cos \left(q_{1}\right) \dot{q}_{1} \dot{q}_{2}^{2} \\
\phi_{21}= & 0 \\
\phi_{22}= & \phi_{12} \\
\phi_{23}= & \cos \left(q_{2}\right) \ddot{v}_{r}+\sin \left(q_{2}\right) \dot{q}_{1} \dot{v}_{r}+\varrho \cos \left(q_{1}+q_{2}\right)
\end{aligned}
$$

where $\varrho=g / r_{1}$, and $g$ is the acceleration of gravity.

The values used in the simulation are selected as $r_{1}=1 \mathrm{~m}$, $r_{2}=0.8 \mathrm{~m}, m_{1}=0.5 \mathrm{~kg}, m_{2}=0.5 \mathrm{~kg}$. Thus, $\rho$ is chosen as $\rho=3$. The parameters $\Lambda$ and $\epsilon$ are chosen as $\Lambda=10$ and $\epsilon=5$. The control gain $K$ and the force control gain $K_{\lambda}$ are chosen as $K=\operatorname{diag}(20,20), K_{\lambda}=200$.

Since the trajectory tracking satisfying the constraint is of interest, the initial positions and velocities of the mechanical system are chosen on desired trajectories, i.e., $q_{1}(0)=0$, $q_{2}(0)=0, \dot{q}_{1}(0)=0, \dot{q}_{2}(0)=\frac{1}{2}$.

Using the control law (26) with (27), the results of the simulation are shown in Figs. 1-6. Fig. 1 shows the trajectory 


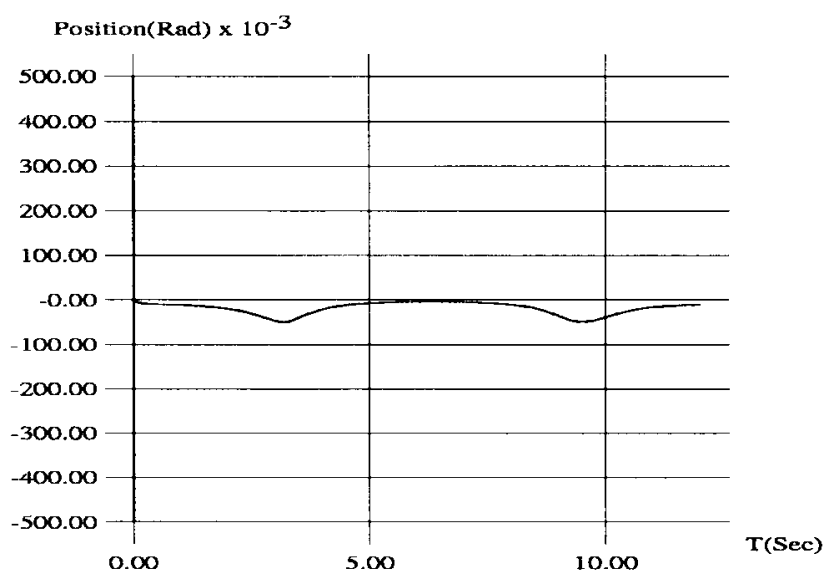

Fig. 1. Position trajectory of $q_{1}$.

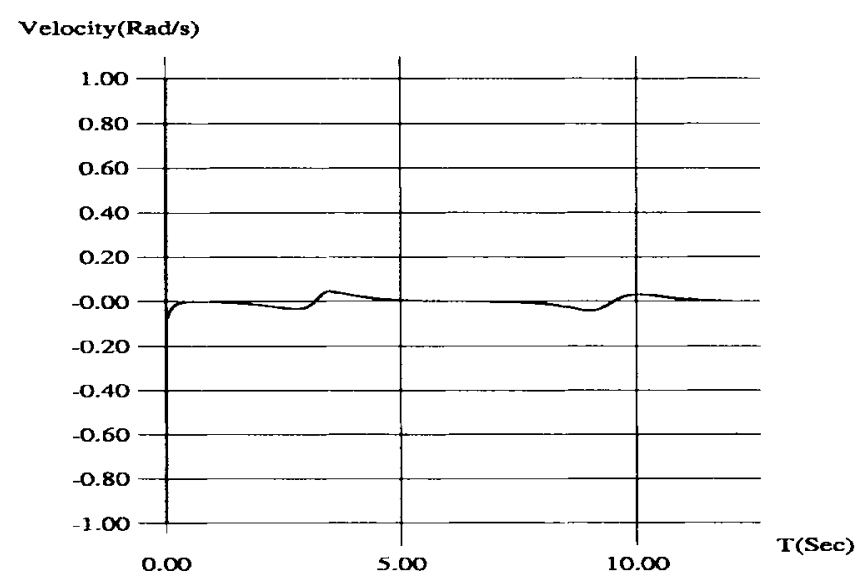

Fig. 2. Velocity of $q_{1}$.

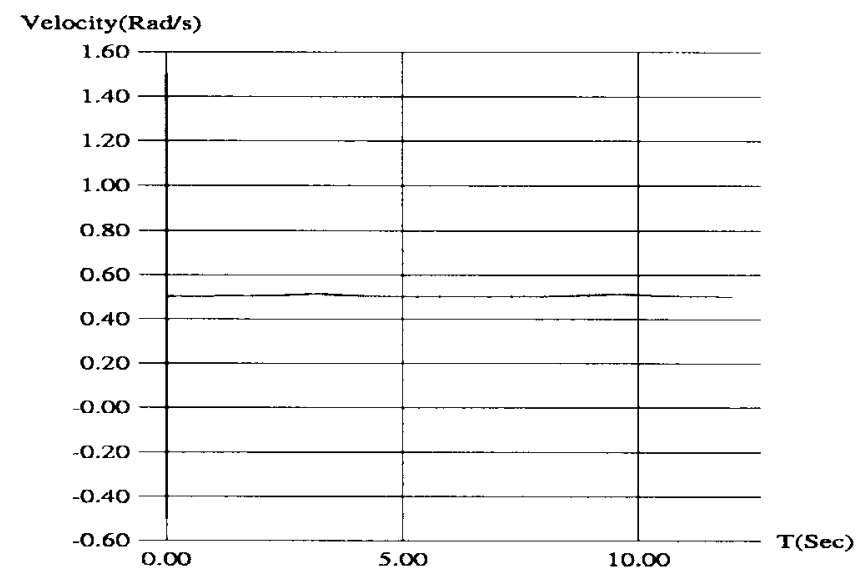

Fig. 3. Velocity of $q_{2}$.

of $q_{1}$, Figs. 2 and 3 show the trajectories of $\dot{q}_{1}$ and $\dot{q}_{2}$, Fig. 4 shows the tracking error of $\lambda$. Figs. 5 and 6 shows the torques exerted on the system. These results show that the control objective is achieved.

\section{CONCLUSION}

In this paper, the issue of appropriate modeling and control is addressed for mechanical systems with Pfaffian nonholo-

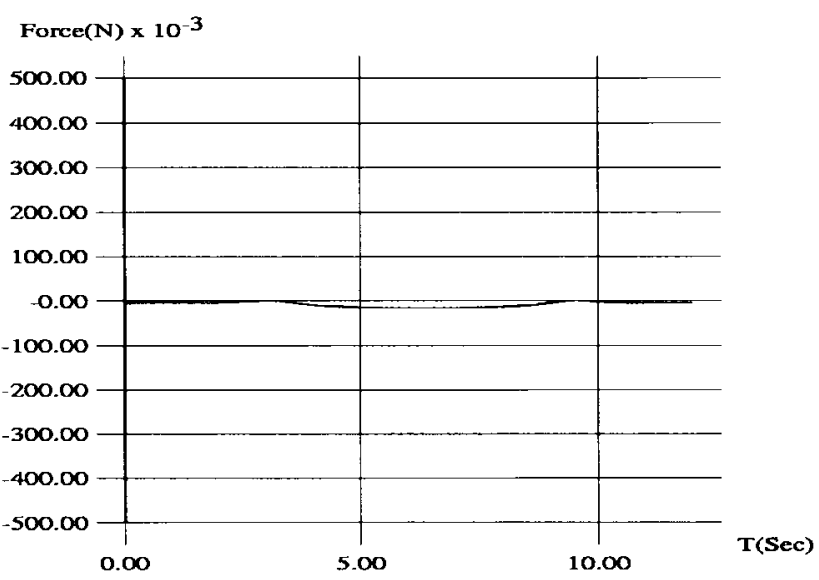

Fig. 4. Contact force error.

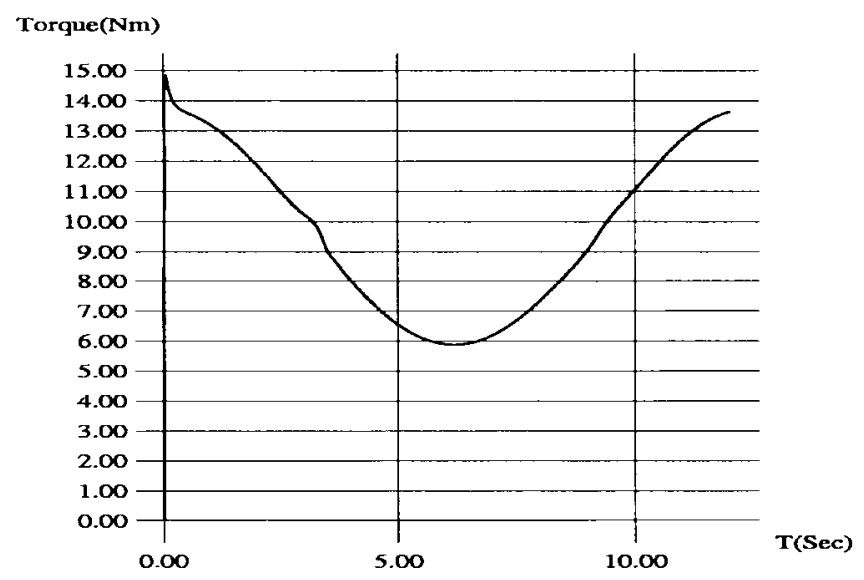

Fig. 5. Input $\tau_{1}$.

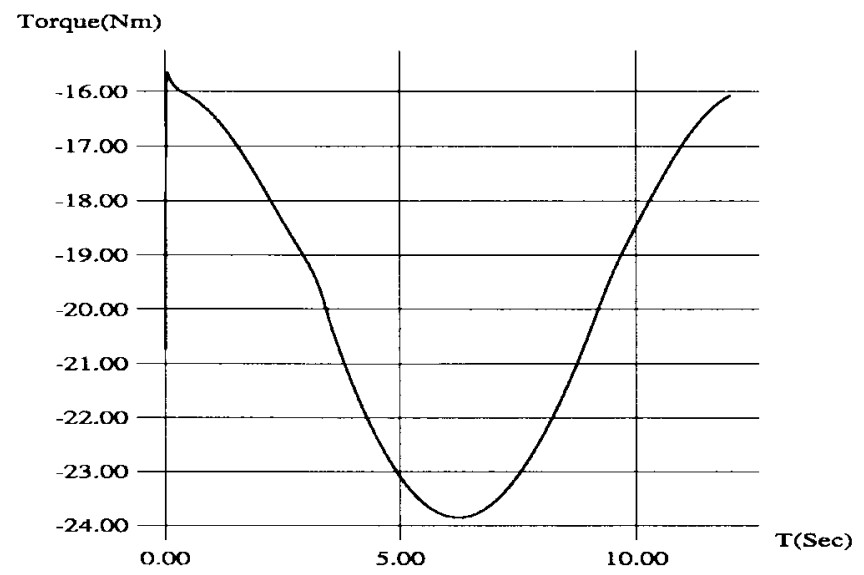

Fig. 6. Input $\tau_{2}$.

nomic constraints. By specifying a pseudo-state vector, a novel dynamic model, suitable for simultaneous force and motion control, is established. A robust control formulations are then proposed, ensuring that a system with $p$ nonholonomic constraints can be stabilized to a $p$-dimensional desired manifold. However, the definition of the desired manifold depends on the specific choice of pseudo-state vector, which is related to the form of the constraint equations. Given the pseudo-state 
vector, the developed method provides a solution for the robust force and motion control of nonholonomic systems. A simple mechanical system with a Pfaffian nonholonomic constraint has been used to illustrate the methodology developed in this paper.

\section{REFERENCES}

[1] A. M. Bloch, M. Reyhanoglu, and N. H. McClamroch, "Control and stabilization of nonholonomic dynamic systems," IEEE Trans. Automat. Contr., vol. 37, pp. 1746-1757, 1992.

[2] G. Campion, B. d'Andrea-Novel, and G. Bastin, "Controllability and state feedback stabilizability of non holonomic mechanical systems," in Advanced Robot Control, C. Canudas de Wit, Ed. Berlin, Germany: Springer-Verlag, 1991, pp. 106-124.

[3] A. M. Bloch, "Stabilizability of nonholonomic control systems," Automatica, vol. 28 , pp. 431-435, 1992.

[4] A. Kapitanovsky, A. A. Goldenberg, and J. K. Mills, "Dynamic control and motion planning technique for a class of nonlinear systems with drift," Syst. Contr. Lett., vol. 21, pp. 363-369, 1993.

[5] R. M. Murray and S. S. Sastry, "Nonholonomic motion planning Steering using sinusoids," IEEE Trans. Automat. Contr., vol. 38, pp. 700-716, 1993.

[6] A. J. Koivo and S. H. Arnautovic, "Control of redundant manipulators with constraints using a reduced order model," Automatica, vol. 30, pp. 665-677, 1994

[7] N. Sarkar, X. Yun, and V. Kumar, "Control of a single robot in a decentralized multi-robot system," in Proc. 1994 IEEE Int. Conf. Robotics Automation, 1994, pp. 896-901.

[8] R. W. Brockett, "Asymptotic stability and feedback stabilization," in Differential Geometric Control Theory, R. W. Brockett, R. S. Millman, and H. J. Sussmann, Eds. New York: Birkhauser, 1983.

[9] Ju. I. Neimark and N. A. Fufaev, "Dynamics of nonholonomic systems," Transl. Math. Monogr., vol. 33, 1972.

[10] R. M. Rosenberg, Analytical Dynamics of Discrete Systems. New York: Plenum, 1977.

[11] A. M. Bloch, P. S. Krishnaprasad, J. E. Marsden, and R. M. Murray, "Nonholonomic mechanical systems with symmetry," Tech. Rep. CDS 94-013, Calif. Inst. Technol., Pasadena, June 1995.

[12] N. Sarkar, X. Yun, and V. Kumar, "Control of contact interactions with acatastatic nonholonomic constraints," Int. J. Robot. Res., vol. 16, pp. 357-374, 1997.

[13] R. E. Kalaba and F. E. Udwadia, "Equations of motion for nonholonomic, constrained dynamical systems via Gauss's principle," J. Appl. Mech., vol. 60, pp. 662-668, 1993.

[14] J. P. Laumond, "Feasible trajectories for mobile robots with kinematic and environment constraints," in Int. Conf. Intelligent Autonomous Systems, 1986, pp. 346-354.

[15] C. Samson, "Velocity and torque feedback control of a nonholonomic cart," in Advanced Robot Control, C. Canudas de Wit, Ed. Berlin, Germany: Springer-Verlag, 1991, pp. 125-151.

[16] J.-M. Corron, "Global asymptotic stabilization for controllable systems without drift," Math. Contr., Signals, Syst., vol. 5, pp. 295-315, 1991.

[17] J.-P. Pomet, "Explicit design of time-varying stabilizing control laws for a class of controllable systems without drift, Syst. Contr. Lett., vol. 18, pp. 147-158, 1992.

[18] C. Canudas de Wit and O. J. Sørdalen, "Exponential stabilization of mobile robots with nonholonomic constraints," IEEE Trans. Automat. Contr., vol. 37, pp. 1791-1797, 1992.

[19] J. B. Pomet, B. Thuilot, G. Bastin, and G. Campion, "A hybrid strategy for the feedback stabilization of nonholonomic mobile robots," in Proc. 1992 IEEE Int. Conf. Robotics Automation, 1992, pp. 129-134.

[20] R. P. Singh and P. W. Likins, "Singular value decomposition for constrained dynamical systems," J. Appl. Mech., vol. 52, pp. 943-948, 1985.

[21] M. W. Spong, "On the robust control of robot manipulators," IEEE Trans. Automat. Contr., vol. 37, pp. 1782-1786, 1992.

[22] G. Oriolo and Y. Nakamura, "Control of mechanical systems with second-order nonholonomic constraints: underactuated manipulators," in Proc. 30th Conf. Decision Control, 1991, pp. 2398-2403.

[23] J. J. E. Slotine and W. Li, "On the adaptive control of robot manipulator," Int. J. Robot. Res., vol. 6, pp. 49-59, 1987.

[24] C.-Y. Su and T.-P. Leung, "A sliding mode controller with bound estimation for robot manipulator," IEEE Trans. Robot. Automat., vol. 9, pp. 208-214, 1993.

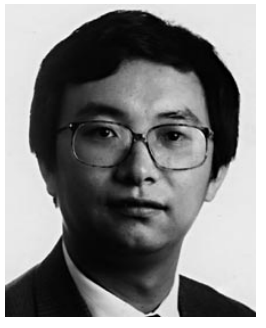

Chun-Yi Su (M'93) was born in Xining, China, in 1962. He received the B.E. degree in control engineering from Shaanxi Institute of Mechanical Engineering (now Xian University of Technology) in 1982, and the M.S. and Ph.D. degrees in control engineering from South China University of Technology in 1987 and 1990, respectively.

From 1990 to 1991, he was with the Department of Automation, South China University of Technology. From 1991 to 1998 , he was with the Department of Mechanical Engineering, University of Victoria, Victoria, B.C., Canada. Since 1998, he has been with Concordia University, Montral, P.Q., Canada, where he is currently Assiatant Professor of mechanical engineering. His main research interests are in robotics, mechatronics, and nonlinear cotrol theory. He is the author of over 80 publications which have appeared in journals, as book chapters, and in conference proceedings.

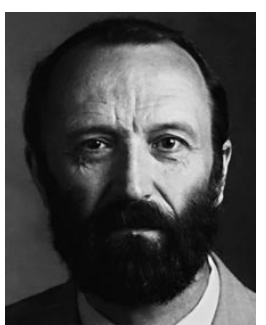

Yury Stepanenko received the Ph.D degree in 1964 from the Moscow Institute of Machine Science, Moscow, Russia, for his work on the analysis of nonlinear servosystems. Working in the area of robotic research, he received the Doctor of Science degree in 1971, from the same university. In pursuing this degree, he originated a few methods of robot dynamic modeling, including the "recursive Newton-Euler approach."

He was Senior Resarcher, Moscow Institute of Machine Science, Profesor and Chairman of the Department of Automatic Control, Institute of Machine-Tool Engineering, Moscow, and Senior Consultant, Iskra Corporation, Slovenia. Since 1982, he has been with a variety of Canadian universities. Currently, he is a Professor, Department of Mechanical Engineering, University of Victoria, Victoria, B.C., Canada, and Director of the university's Adaptive Robotic Telesystems Laboratory. He is the author and co-author of more then 120 scientific papers and a monograph entitled "Dynamics of spatial mechanisms." $\mathrm{He}$ also holds ten patents. His research interests are in dynamics and control of nonlinear systems and robotics.

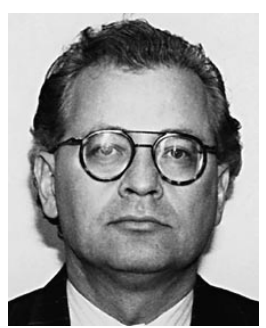

A. A. Goldenberg (S'73-M'76-SM'87-F'96) received the B.A.Sc. and M.A.Sc. degrees from Technion-Israel Institute of Technology, Haifa, in 1969 and 1972, respectively, and the Ph.D. degree from the University of Toronto, Toronto, Ont., Canada, in 1976, all in electrical engineering.

From 1975 to 1981, he was with Spar Aerospace Ltd., Toronto, where he worked mainly on control, analysis, and design of the space shuttle remote manipulator system and satellite controls. From 1981 to 1982, he was an Assistant Professor of Electrical Engineering and from 1982 to 1987, he was an Associate Professor of Mechanical Engineering, University of Toronto. Since 1987, he has been a Professor of mechanical engineering. He holds cross appointments in the Department of Electrical Engineering and the Institute of Biomedical Engineering. He founded the Robotics and Automation Laboratory, University of Toronto, in 1982 and the Mechatronics Laboratory, University of Toronto, in 1996. His current research interests are in the field of robotics and industrial automation, kinematics, control and dynamics of robots, and dexterous endeffectors. He is the founder and President of Engineering Services, Inc., a high-technology company involved in the development of prototype roboticbased automation, and products. He is a consultant to IBM of Canada Ltd., Department of National Defence, Ontario Ministry of Agriculture and Food, RCMP, Consumers Gas, Vulcan-Vulcap Inc., and DCIEM.

Dr. Goldenberg is Editor of the IEEE Transactions on Robotics AND Automation. He a member of the American Society of Mechanical Engineers and the Professional Engineers of Ontario. 